\title{
Hormonal control of root growth and development in ABA deficient barley mutant
}

\author{
Veselov D.*, Vysotskaya L., Timergalina L., Fedyaev V. \\ Ufa Institute of Biology, UFRC RAS, Ufa, Russia \\ *email:veselov@anrb.ru
}

Phytohormone abscisic acid (ABA) plays important role in the control of plant development. Its accumulation under stress conditions is involved in plant adaptation to changing environment. Genetic control of ABA synthesis is thoroughly studied and mutations of the genes responsible for the process have been shown to lead to the hormone deficiency in planta. We worked with barley mutant AZ34 with impaired function of the molybdenum cofactor of the enzyme catalyzing oxidation of ABA aldehyde. Its comparison with the parental barley cultivar Steptoe allowed revealing the importance of this hormone for regulation of root growth and development. We studied the effects of over-optimal concentrations of mineral nutrients resulting in mild osmotic stress on root growth and cytokinin concentration in plants of both genotypes. Interest to cytokinins was dictated by information on importance of interaction between cytokinins and $\mathrm{ABA}$ for the control of plants responses to availability of mineral nutrients. Increased concentration of mineral nutrients resulted in inhibition of root elongation accompanied by accumulation of cytokinins in roots of Steptoe plants. Root growth response was likely to be due to accumulation of cytokinins detected in the stressed plants, since these hormones are known to inhibit root growth. Both hormonal and growth response were absent in the ABA deficient mutant suggesting importance of capacity of the plants to accumulated ABA for the control of cytokinin concentration in the roots and inhibition of their elongation under mild osmotic stress. Accumulation of cytokinins in the roots of Steptoe was accompanied by a decline in their concentration in the shoots suggesting inhibition of cytokinin export from root to shoots under osmotic stress caused by excessive concentration of mineral nutrients. We hypothesize that detected changes in cytokinin distribution between roots and shoots are due to ABAinduced inhibition of cytokinin transport from the roots.

Acknowledgments: The work was supported by grant No. 20-04-00305 of RFBR. 